\title{
Evaluación del impacto de los cambios de las metodologías de enseñanza-aprendizaje en egresados de los programas de Medicina y
} Enfermería de la Universidad del Norte, Colombia

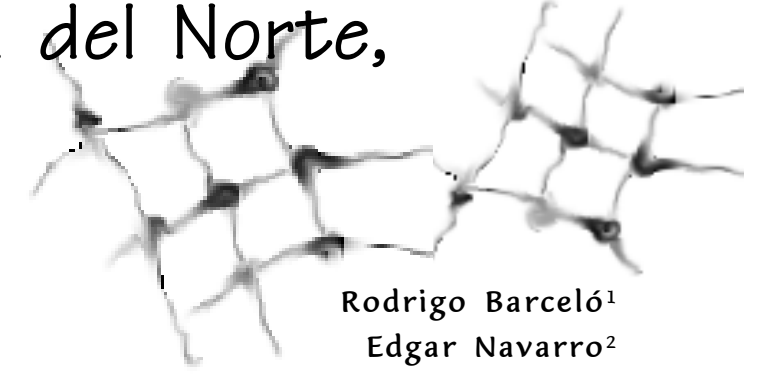

BARCELÓ, R.; NAVARRO, E. Assessment of the effect of the exchange rate on the teaching-learning methods deriving from the Medicine and Nursing programs of the Universidad del Norte, Colombia, Interface Comunic, Saúde, Educ, v.7, n.13, p.65-78, 2003.

A transverse descriptive study was carried out in order to assess the effect of the changes to the teaching learning methods of students of the Medicine and Nursing programs of the Universidad del Norte of Barranquilla, Colombia. Groups of students graduating in 1993 and 1999 were compared. The latter correspond to the first group of graduates exposed to the changes in the curricula promoted by the Barranquilla UNI Project. The information was obtained by means of a questionnaire addressed to those taking part. The results show statistically significant differences between the groups, favoring the UNI - exposed graduate groups using active student-centered teaching methods, the ability to gain access to new technologies, fostering cross-professional and community teamwork, continuing self-learning after graduation, conducting research into the epidemiological circumstances of the regions and proposing solutions to the needs expressed by the community. As these students have very recently graduated, new studies should be carried out for subsequent assessment and monitoring.

KEY WORDS: Evaluation studies; teaching; learning; methods; curriculum; universities; Medical Education.

Se realizó un estudio descriptivo transversal con el objetivo de evaluar el impacto de los cambios en las metodologías de enseñanza-aprendizaje en estudiantes de los programas de Medicina y Enfermería de la Universidad del Norte de Barranquilla, Colombia. Se compararon dos cohortes de estudiantes egresados en los años 1993 y 1999, esta última correspondiente al primer grupo de egresados expuestos a la influencia de los cambios curriculares propiciados por Proyecto UNI - Barranquilla. La información se obtuvo mediante la aplicación de una encuesta autodiligenciada a los participantes. Los resultados muestran diferencias estadísticamente significativas entre los grupos a favor de la cohorte de egresados expuestos al ideario UNI respecto a: utilización de metodologías activas de enseñanza, posibilidad de utilizar nuevas tecnologías, implementar el trabajo multiprofesional y con líderes comunitarios, dar continuidad al autoestudio, investigar la realidad epidemiológica de la región y proponer soluciones para las necesidades presentadas por la comunidad. Como el tiempo de formación de esos estudiantes es muy reciente, deberán ser realizados nuevos estudios para evaluación y seguimiento posterior.

PALABRAS CLAVE: Estudios de evaluación; enseñanza; aprendizaje; metodos; currículo; universidades; Educación Médica.

\footnotetext{
${ }^{1}$ Director do Proyecto UNI - Barranquilla; Docente, Departamento de Salud Familiar y Comunitaria. Universidad del Norte. <rbarcelo@uninorte.edu.co>

${ }^{2}$ Coordinador de Investigación. Proyecto UNI - Barranquilla. Docente, Departamento de Salud Familiar y Comunitaria. Universidad del Norte.<enavarro@uninorte.edu.co>
} 


\section{Introducción}

Las características del entorno y los cambios permanentes que ocurren en nuestra sociedad influyen de manera decisiva en la educación superior, y por lo tanto esta debe realizar los ajustes necesarios en los currículos de los programas para dar respuesta a los requerimientos y necesidades que aparecen y así cumplir cabalmente su papel social. La práctica médica y de enfermería no esta exenta de la realidad social y por lo tanto deben realizar los ajustes necesarios para adaptarse a los cambios.

Los cambios han ocurrido en diferentes aspectos, dentro de los cuales se encuentran: la atención del paciente (se transformó la práctica individual en una práctica colectiva; lo anterior conduce a establecer la necesidad de entender al individuo dentro del contexto familiar y social; los cambios ocurridos en la legislación de la seguridad social que exigen una prestación de servicios médicos eficientes, de buena calidad y con una amplia cobertura; énfasis en los aspectos de promoción de la salud y prevención de la enfermedad); en la ciencia y tecnología (la producción vertiginosa de información científica y su amplia difusión por redes computarizadas; innovaciones en los campos de la genética y la inmunología; avances en la biotecnología); en la estructura demográfica (inversión en la relación urbano/rural en el país debido a los problemas sociales, económicos y de violencia; inversión de la pirámide poblacional con disminución de los grupos de población de 0 a 15 años y aumento del grupo de edad entre 15 a 64 años, y leve incremento en los mayores de 65 años) (Velasco, 1993); en la legislación (en la constitución colombiana de 1991 se establece que la salud es un derecho irrenunciable del individuo; las modificaciones establecidas por la Ley 100 de 1993; la ley 60 de 1993 sobre la distribución de competencias y recursos; incremento de la participación ciudadana en la toma de decisiones en el Sector Salud); en la tendencia epidemiológica (los cambios en la estructura demográfica y social del país conllevan a una transición epidemiológica) (Velasco, 1993).

Pero a pesar de que se aprecian en los primeros lugares de la morbilidad y mortalidad patologías propias de países desarrollados existe aún presencia de patologías tales como paludismo, parasitismo, desnutrición, tuberculosis, consideradas como enfermedades reemergentes (Ordóñez, 1995).

En cuanto a las causas de morbilidad de consulta externa y egresos hospitalarios el primer lugar es ocupado por embarazo y parto normal y sus complicaciones, en la consulta de urgencia este lugar lo ocupan los traumas $y$ heridas como consecuencia de la etapa de violencia social que atraviesa el país (Ordóñez, 1995).

Al realizar el análisis de los años de vida potencialmente perdidos y años de vida saludables perdidos (AVISAS) (Murray, 1995) se aprecia que las patologías relacionadas con la violencia también ocupan el primer lugar en nuestro país, seguidas de las enfermedades cardiovasculares e infecciosas (Corposalud, 1997).

Por todo lo anterior, es necesario realizar en los currículos de Medicina y Enfermería cambios que conlleven a una estrategia de articulación de la 
enseñanza del aprendizaje que trascienda el trabajo de una sola profesión e involucre la conformación de equipos multiprofesionales que desde el pregrado le permitan al estudiante trabajar en equipo con otras profesiones respetando cada uno su campo de saberes y donde la comunidad se involucre no solo como receptora de actividades de los servicios y la academia sino como participante activa en todas las acciones sobre ellos emprendidas.

Con el anterior ideario, se inició el Proyecto UNI - Barranquilla, Una Nueva Iniciativa en la Educación de los Profesionales de la Salud, proyecto de ámbito comunitario en el cual participan como socios desde el inicio, agosto de 1994, La Universidad del Norte, La Comunidad de trece barrios del Suroccidente de Barranquilla, Distrisalud, Dasalud y Organizaciones No Gubernamentales (Fe y Alegría, Plan Internacionales) además se ha integrado al proceso la Universidad del Atlántico, con su Facultad de Nutrición y Dietética.

Las estrategias que utiliza el Proyecto son la integración vertical y horizontal para articular los esfuerzos de los diversos departamentos $y$ disciplinas en el desarrollo de los currículos de Medicina y Enfermería de la Universidad del Norte. El mercadeo social que permite identificar, anticipar y satisfacer las necesidades y expectativas de la comunidad. La Planificación Estratégica nos ayuda a identificar las fortalezas, debilidades, oportunidades y amenazas en cada uno de los componentes del Proyecto, además de establecer prioridades y planes de desarrollo institucional. Con la Investigación Acción se realiza el diagnóstico de los problemas de la comunidad, servicios y universidad; la Metodología de Sistemas como enfoque sistemático en el análisis de problemas contribuye al desarrollo de la evaluación de cada uno de los componentes del Proyecto (Oficina de Planeación Universidad del Norte, 1995).

El proyecto UNI presenta como resultado esperado en la academia el establecimiento de modelos académicos de unión con la comunidad y articulación con los servicios de salud, que incluyan: tecnologías apropiadas para enseñanza-aprendizaje junto con la presentación de servicios en centros de salud, o junto a la comunidad en equipos multiprofesionales; ajustes académicos necesarios para que el trabajo antes mencionado sea realizado en tiempo curricular; actividades de investigación clínica, epidemiológica y gerencial orientada a la solución de problemas de la comunidad y de los servicios; desarrollo de nuevos líderes en el campo de la educación de los profesionales de la salud (Kisil \& Chaves, 1994).

El componente de Evaluación, dentro del Proyecto UNI Barranquilla, es el encargado de identificar, obtener y proporcionar información útil descriptiva acerca del valor y el mérito de las metas, la planificación, la realización y el impacto de las actividades y procesos desarrollados por los otros componentes del Proyecto con el fin de servir de guía para la toma oportuna de decisiones, solucionar los problemas y promover la comprensión de los fenómenos implicados (Jauregui \& Suarez Chavarro, 1998; Barcelo et al., 1996; Aguilar \& Ander-Egg, 1994).

En la segunda fase, en la cual se encuentra el Proyecto UNI Barranquilla, el componente Evaluación centra sus actividades en identificar y determinar 
el resultado e impacto de los procesos iniciados durante la primera fase. Dentro de la determinación del resultado e impacto de los cambios surgidos en la academia está evaluar el desempeño de los estudiantes egresados de los programas de medicina y enfermería antes del inicio del proyecto UNI Barranquilla para realizar la comparación con el desempeño de la primera cohorte de estudiantes que recibieron todos los cambios surgidos en los currículos y metodologías de enseñanza-aprendizaje por influencia del proyecto UNI.

Los resultados de la presente investigación contribuirán de manera importante en la evaluación de Cluster realizada por la Fundación W. K. Kellogg.

\section{Evolución del currículo de la Facultad de Medicina} La Universidad del Norte en los inicios de la Facultad de Medicina, se planificó para tener como producto final del currículo a un médico general bien informado y de excelente formación ética y moral. Era un currículo rígido, con una fijación vertical y horizontal inamovible, en el que no se observaba una proyección a la comunidad, que estudiara y enseñara a los alumnos las prioridades de los problemas de salud tanto en la comunidad como en la región y el país (Espinosa Taboada, 1997).

Era la forma universal de enfocar la salud, y las escuelas de medicina aunaban todo su esfuerzo en formar profesionales cada día más expertos en curar la enfermedad y desarrollar tecnologías más capaces de diagnosticarla. Sin embargo, era preocupante ya en las organizaciones de salud a nivel mundial el incremento cada día más evidente de las enfermedades tanto en el campo infeccioso como en el crónico. Se investigaba muy aceleradamente para conseguir un marco que permitiera campañas y educación tanto en la epidemiología como en la prevención de las enfermedades. Todo ello provocó que en las facultades de medicina, inspiradas por las organizaciones mundiales de la salud y los encuentros a nivel de los países y del mundo en general, se iniciara una preocupación constante para buscar, aplicar y poder desarrollar un currículo que permitiera no sólo conocer las enfermedades de la comunidad, sino enfocar los problemas de salud hacia las prioridades en estas enfermedades, $y$ en forma activa poder educar a los nuevos galenos con una proyección eminentemente social y comunitaria.

Fue así como la División Ciencias de la Salud propuso a las autoridades de la Universidad una reforma curricular para ser aplicada a partir de 1988, y que fundamentalmente incluyera cambios metodológicos en la enseñanza y en la proyección del aprendizaje de los alumnos hacia la comunidad.

Basado en lo anterior, en 1986 se inició una revisión exhaustiva de todo el currículo, el contenido de sus cátedras, la aplicación de los contenidos en las distintas etapas y la repetición de muchos elementos en la enseñanza, y finalmente el tiempo requerido para un aprovechamiento de los alumnos en las distintas cátedras.

Esta investigación se llevó a cabo con participación de alumnos, profesores, coordinadores de cátedra y jefes de departamento. Después de varias reuniones de distinto tipo, y dirigidos por el Comité Curricular, y de análisis de contenido, se llegó a la conclusión, conforme a la época, que era 
necesario aumentar un año más al currículum, con el fin de satisfacer las enseñanzas a nivel de Medicina Interna, Cirugía General y Especialidades Quirúrgicas, además de insertar nuevas cátedras que cubrieran las necesidades de enseñanza a los alumnos, como son la Salud Ocupacional y la Gerencia en Salud. Igualmente se consideró que los alumnos debían presentar un trabajo científico, ubicado en el VIII y XII semestre, con lo cual se satisfizo el desarrollo de la investigación, como eje fundamental del nuevo currículum.

Con este currículum se inició en forma tímida una integración entre los distintos Departamentos de la División y sus respectivas cátedras, de tal manera que docentes de Medicina Preventiva se involucran en la enseñanza de Ciencias Básicas. También, docentes de las Ciencias Clínicas Médicas alternan en algunas cátedras con docentes de los otros departamentos.

Concluidos todos los estudios y análisis para esta reforma curricular, finalmente es aprobada por el Comité Curricular y a nivel del Consejo Académico, y se escogió para su iniciación el primer semestre de 1988. Con este currículum se lograron los siguientes propósitos:

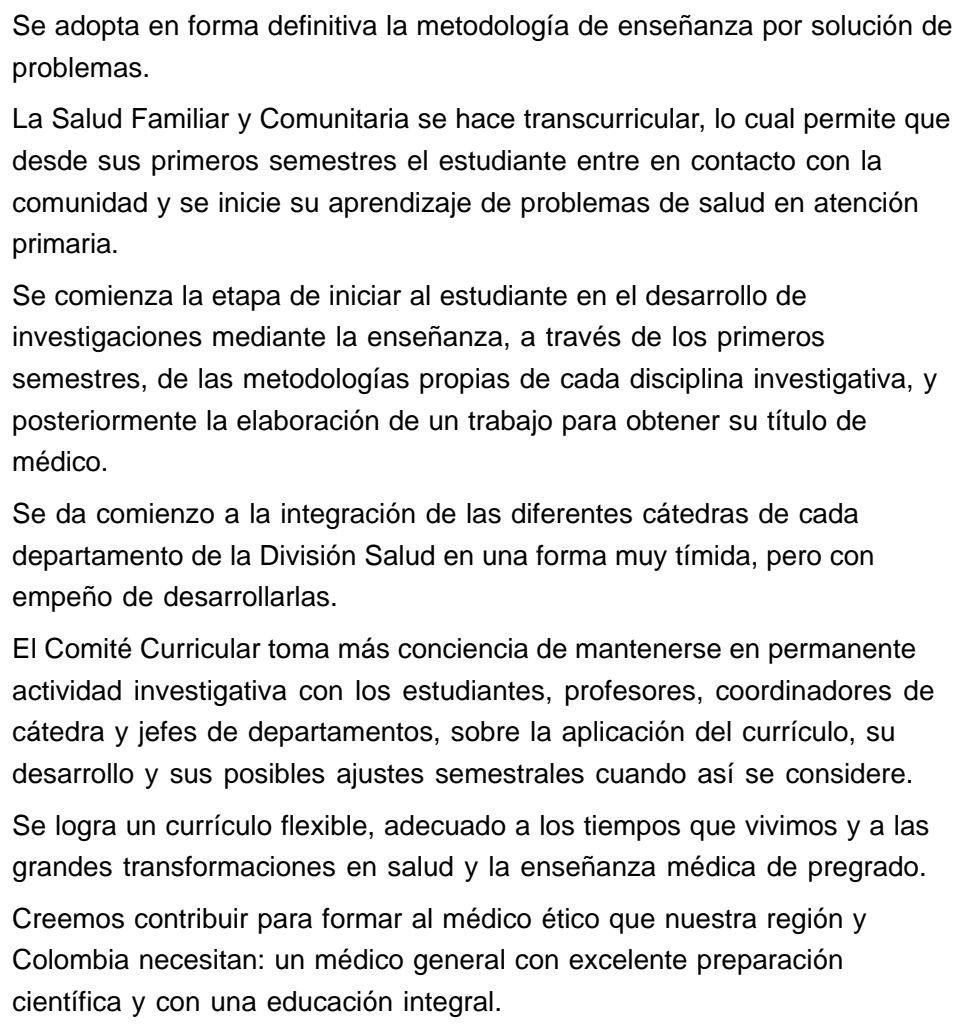

Hasta este momento hemos analizado lo que ha pasado con nuestro currículo después de quince años de evolución de nuestra Facultad de Medicina.

Es indudable el progreso tanto en la enseñanza científica como en la formación humanística que se les brindará a nuestros estudiantes a partir de esta reforma. Así mismo, se consideró que la permanente evaluación en 
el desarrollo del currículo exigía ajustes e innovaciones que debían hacerse en cada semestre, acordes con la evolución en la enseñanza médica y las reformas en el campo de la seguridad social que estaban por llevarse a cabo en el país.

A partir de 1993 se consideró la imperiosa necesidad de iniciar una integración Básico-Clínica - Preventiva y Comunitaria, para lo cual se llevó a cabo un análisis del contenido de cada programa y la elaboración de seminarios y paneles cuyo contenido tuviera el marco de clases magistrales, con el objeto de que a medida que se desarrollara la enseñanza por solución de problemas se fueran suprimiendo paulatinamente las clases magistrales en cada programa.

A principios de 1994, la Rectoría planificó, para toda la Universidad, hacer una Reconceptualización del Crédito, y poder así evaluar el tiempo necesario para cada asignatura y considerar el espacio que cada estudiante debiera tener para sus propias actividades en sus obligaciones académicas.

Fue así como el Comité Curricular, en forma muy activa, procuró un intercambio de los contenidos de cada programa entre los distintos departamentos y cada una de las cátedras.

A partir de 1994 con el inicio del proyecto UNI - Barranquilla se incrementa más la integración entre los distintos departamentos, y se establecen las bases para lograr una mayor aplicación, entre alumnos y profesores, de la Metodología de enseñanza por solución de problemas y el aprendizaje basado en problemas (Espinosa Taboada, 1997).

Con todos estos sucesos y haciendo el Comité Curricular permanentes evaluaciones del proceso de enseñanza y aplicaciones y desarrollo del currículo, se propuso, para iniciar en el II semestre de 1995, una integración entre las cátedras del área del Comportamiento y Salud Mental, con las cátedras de Salud Familiar y Comunitaria, y Química y Biología y Ciencias Básicas. Fue así como la Psicología Medica, Psicología Evolutiva y Psicopatología se integraron con las cátedras de Salud Familiar y Comunitaria I, II, III, IV y V. Además, en Psiquiatría, aunque quedó como una cátedra aparte, se involucraron Módulos en Medicina Interna I, Medicina Interna II, Ginecología y Obstetricia y Cirugía I.

En forma simultánea se inició Historia de la Medicina en el segundo semestre, integrada a Morfofisiología, y en el tercer semestre a Morfología, y se suprimió en el primer semestre. Se trasladó la Introducción a la Salud Familiar y Comunitaria al primer semestre. Creemos que de esta manera se logre preparar en forma aún más práctica a los nuevos alumnos para la actividad temprana de la medicina comunitaria, reforzada con la enseñanza que se da en la Introducción a la vida universitaria, directamente por la Dirección de Programas, sobre inducción de las metodologías de la enseñanza por solución de problemas y el aprendizaje basado en problemas.

Dentro de los cambios más relevantes ocurridos con el Proyecto UNI Barranquilla en los currículos de Medicina y Enfermería se encuentran: orientado hacia la comunidad, trabajando de manera integrada con miembros de la comunidad y no por o para la comunidad; integración horizontal de los contenidos (clínico - básico - epidemiológica) tanto en la teoría como en la práctica; investigación como transdisciplina; basada en la 
Atención Primaria en Salud; el estudiante como eje del proceso de aprendizaje; trabajo en equipos multiprofesionales desde los primeros semestres de su formación; practica clínica descentralizada de los niveles terciarios de atención a los centros de salud del primer nivel de atención; empleo de metodologías activas de aprendizaje, en aproximadamente el $70 \%$ de las asignaturas del Plan de estudio, que incluyen solución de problemas, aprendizaje basado en problemas, aprendiendo a aprender e investigación acción - participación.

La División Ciencias de la Salud de la Universidad del Norte con sus programas de Medicina y Enfermería ha participado desde el inicio del Proyecto UNI - Barranquilla, en 1994, por lo que se considera importante evaluar el desempeño de dos cohortes de estudiantes egresados de los programas de Medicina y Enfermería, las cohortes del 93 y 99, sin y con la influencia de las modificaciones realizadas en las metodologías de enseñanza-aprendizaje comparado para evaluar de manera indirecta los resultados e impacto debido al proyecto su desempeño.

\section{Objetivos}

Evaluar el impacto de los cambios en las metodologías de enseñanzaaprendizaje en estudiantes de los programas de Medicina y Enfermería de la Universidad del Norte;

Recibir información eficiente de los egresados para la evaluación institucional y los ajustes curriculares;

Realizar los ajustes institucionales necesarios para la promoción de profesionales con mayor capacidad para el ejercicio de sus labores, con espíritu investigativo y conocimiento de los campos ocupacionales en que se va a desempeñar.

\section{Materiales y métodos}

Un estudio descriptivo trasversal (Pineda et al., 1994), en el cual no se busca establecer una representación probalística del Universo de egresados sino establecer una comparación entre una cohorte de egresados que no hayan recibido la influencia de los cambios generados por el Proyecto UNI Barranquilla en los currículos de Medicina y Enfermería (cohorte 1993) y comparado con una cohorte que recibió la influencia del Proyecto UNI Barranquilla (cohorte 1999).

Población de estudio: el proceso de selección de la muestra no se realizará para ninguno de los egresados de los dos programas de manera aleatoria porque no se pretende una representación de todo el Universo sino extraer información de los actores claves dentro del proceso.

La muestra estuvo constituida por 65 egresados de los programas de medicina y enfermería de ambas cohortes.

La unidad de análisis estuvo constituida por los egresados de los programas de Medicina y Enfermería que brinden la información solicitada en el cuestionario de recolección de la información.

Recolección de la información: la información se recolecta mediante una encuesta autodiligenciada en la cual se obtuvieron los datos de identificación de los egresados, problemas surgidos durante la carrera, 
realización de prácticas en la comunidad y/o puestos de salud, fuentes de obtención de información para su aprendizaje, concepciones y opiniones sobre: los cambios o innovaciones en la educación, los procesos de evaluación utilizados en las asignaturas del plan de estudio, la participación de la comunidad en la toma de decisiones relacionadas con la salud, el trabajo en equipo multiprofesional y la utilidad de los procesos de investigación en la generación de conocimiento científico durante su desempeño profesional.

La recolección de la información se lleva a cabo a través de dos métodos, primero de encuestas a los egresados que se encuentren en la ciudad de Barranquilla o en ciudades próximas a la ciudad de Barranquilla. Otra parte de los egresados fue localizada a través de llamadas telefónicas y envío posterior de cuestionario por correo postal certificado.

Para la realización de las encuestas se realizaron las siguientes etapas: estandarización de encuestadores, con la finalidad de establecer una recolección adecuada de la información se realizó el entrenamiento de un grupo de encuestadores en el diligenciamiento del formulario y realización de Prueba Piloto.

Posteriormente se revisaron las recomendaciones y observaciones realizadas por los encuestadores y encuestados para realizar los ajustes necesarios y reproducir el cuestionario de la investigación.

El procesamiento de los datos se realizó con una base de datos simple creada en EpiInfo v. 6.04c.

$\mathrm{El}$ análisis de los resultados se realizó utilizando medidas de frecuencias relativas para aquellas variables de tipo cualitativo y medidas de tendencia central (promedio) y de dispersión (desviación estándar) para las de tipo cuantitativo que fueron recogidas en nivel razón. Para las variables de tipo cuantitativo recogidas en rangos se realizó un análisis con frecuencias relativas.

El análisis de los resultados se ejecutó de manera global para los egresados del programa de Medicina y Enfermería. No se realizó análisis en el interior de cada uno de los grupos debido a que el tamaño de la muestra de manera individual no permite la inferencia adecuada de los resultados a la población de la cual proviene cada uno de ellos.

\section{Resultados}

En la tabla 1, se aprecia que no existen diferencias estadísticamente significativas entre las características sociodemográficas y con su desempeño durante las carreras en las cohortes de estudiantes estudiadas. El promedio de edad, el porcentaje de hombres y el porcentaje de personas casadas o en unión libre en las cohortes es similar. De igual manera los grupos son homogéneos en cuanto a promedio de años de duración de la carrera tanto en el programa de medicina como de enfermería y el promedio académico acumulado (rango de valores posibles de 1-5).

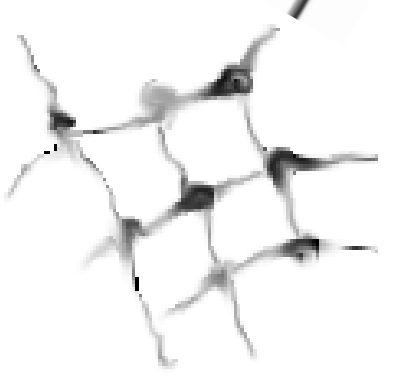


EVALUACIÓN DEL IMPACTO DE LOS CAMBIOS ...

Tabla 1. Distribución de características sociodemográficas de las cohortes de estudiantes de medicina y enfermería.1993 - 1999. Universidad del Norte. Barranquilla

\begin{tabular}{|c|c|c|c|c|}
\hline Característica & $\begin{array}{c}\text { Cohorte } \\
1993\end{array}$ & $\begin{array}{c}\text { Cohorte } \\
1999\end{array}$ & Prueba estadística & Valor de $p$ \\
\hline $\begin{array}{l}\text { Promedio de años de duración de la } \\
\text { carrera de Medicina }\end{array}$ & 6.2 & 6.1 & Prueba $t=0.59$ & 0.55 \\
\hline $\begin{array}{l}\text { Promedio de años de duración de la } \\
\text { carrera de Enfermería }\end{array}$ & 4.3 & 4.3 & Prueba $t=0.1$ & 0.957 \\
\hline Edad & 24.5 & 23.8 & Prueba $t=1.89$ & 0.06 \\
\hline Promedio académico acumulado & 3.73 & 3.84 & Prueba $t=0.93$ & 0.35 \\
\hline Sexo (porcentaje de hombres) & 68 & 64 & Chi cuadrado $=0.14$ & 0.71 \\
\hline $\begin{array}{l}\text { Estado civil durante la carrera (\% de } \\
\text { casados o unión libre) }\end{array}$ & 3 & 5 & Chi cuadrado $=0.01$ & 0.93 \\
\hline
\end{tabular}

En la tabla 2, se muestran las características que los egresados consideraron predominantes en las currículos cursados dentro de su formación profesional. Los datos muestran diferencias estadísticamente significativas a un nivel del $95 \%$ a favor de la cohorte del 99 , en cuanto al grado de utilización de metodologías de enseñanza centradas en el estudiante, la posibilidad de acceso a nuevas tecnologías para el fortalecimiento del proceso de aprendizaje, el fomento del trabajo en equipo para asegurar los problemas de la comunidad y el fortalecimiento del aprendizaje autodirigido y para continuar aprendiendo después de la graduación.

Tabla 2. Distribución de aspectos curriculares en las cohortes de estudiantes de Medicina y Enfermería.1993-1999. Universidad del Norte. Barranquilla

\begin{tabular}{|c|c|c|c|c|c|c|}
\hline \multirow{2}{*}{ Aspectos evaluados } & \multicolumn{2}{|c|}{ Cohorte 1993} & \multicolumn{2}{|c|}{ Cohorte 1999} & \multirow{2}{*}{ Chi cuadrado } & \multirow{2}{*}{ Valor de $p$} \\
\hline & SI & No & SI & NO & & \\
\hline $\begin{array}{l}\text { Utilización de metodologías de enseñanza } \\
\text { centradas en el estudiante }\end{array}$ & 12 & 53 & 61 & 4 & 75.44 * & 0.0000001 \\
\hline $\begin{array}{l}\text { Possibilidad de acceso a nuevas } \\
\text { tecnologías para el fortalecimiento del } \\
\text { proceso de aprendizaje }\end{array}$ & 15 & 50 & 58 & 7 & $57.32^{*}$ & 0.0000001 \\
\hline $\begin{array}{l}\text { Fomento del trabajo en equipe para } \\
\text { asegurar los problemas de la comunidad }\end{array}$ & 18 & 47 & 53 & 12 & $37.32^{*}$ & 0.000001 \\
\hline $\begin{array}{l}\text { Fortalecimiento del aprendizaje } \\
\text { autodirigido y para continuar aprendiendo } \\
\text { después de la graduación }\end{array}$ & 39 & 26 & 59 & 6 & $16.45^{*}$ & 0.0000498 \\
\hline
\end{tabular}

* Estadísticamente significativo a un $95 \%$

En la tabla 3, se puede apreciar que existen diferencias estadísticamente significativas a un nivel del $95 \%$ en cuanto a los procesos académicos desarrollados por los egresados de la cohorte del 99, entre los que se resaltan la existencia de trabajo conjunto con lideres comunitarios, la realización de prácticas en centros comunitarios y primer nivel de atención en salud, la participación de los profesionales de los centros de salud y de la 
BARCELÓ, R.; NAVARRO, E.

comunidad en el proceso de enseñanza de estudiantes y el desarrollo de investigaciones teniendo en cuenta la realidad epidemiológica de la región.

Tabla 3. Distribución de aspectos curriculares en las cohortes de estudiantes de medicina y enfermería.1993-1999. Universidad del Norte. Barranquilla

\begin{tabular}{|l|c|c|c|c|c|c|}
\hline \multicolumn{1}{|c|}{ Aspectos evaluados } & \multicolumn{2}{c|}{ Cohorte 1993 } & \multicolumn{2}{c|}{ Cohorte 1999 } & \multirow{2}{*}{ Chi cuadrado } & Valor de $\mathbf{p}$ \\
\cline { 2 - 8 } & SI & NO & SI & NO & \\
\hline $\begin{array}{l}\text { Existencia de trabajo conjunto con lideres } \\
\text { comunitarios }\end{array}$ & 23 & 42 & 54 & 11 & $\mathbf{3 0 . 3 8 ^ { * }}$ & $\mathbf{0 . 0 0 0 0 0 0 1}$ \\
\hline $\begin{array}{l}\text { Prácticas en centros comunitarios y } \\
\text { primer nivel de atención en salud }\end{array}$ & 43 & 22 & 62 & 3 & $17.74^{*}$ & $\mathbf{0 . 0 0 0 0 2 5 3}$ \\
\hline $\begin{array}{l}\text { Professionales de los centros de salud y } \\
\text { de la comunidad participando en el } \\
\text { proceso de enseñanza de estudiantes }\end{array}$ & 37 & 38 & 61 & 4 & $23.69^{*}$ & $\mathbf{0 . 0 0 0 0 0 1 1}$ \\
\hline $\begin{array}{l}\text { Investigaciones realizadas teniendo en } \\
\text { cuenta la realidad epidemiológica de la } \\
\text { región }\end{array}$ & 22 & 43 & 57 & 8 & $39.22^{*}$ & $\mathbf{0 . 0 0 0 0 0 0 1}$ \\
\hline
\end{tabular}

${ }^{*}$ Estadísticamente significativo a un $95 \%$

En la tabla 4, se destacan los aspectos dentro de los currículos de los programas que no muestran diferencias estadísticamente significativas entre las cohortes de estudio. Dentro de ellas se encuentran: la evaluación de los estudiantes centrada en procesos más que en resultados, una posible explicación de este hecho es que a pesar de existir un incremento significativo en las metodologías de enseñanza-aprendizaje activas no se ha podido lograr una modificación en el esquema de evaluación el cual se encuentra centralizado en la Universidad y es igual para todas las otras carreras diferentes a las de salud. Otro de los aspectos que no mostró diferencias significativas en las cohortes fue el fortalecimiento de los aspectos éticos en el proceso de atención en las instituciones de salud y en la interacción con los pacientes, pero el cual no se puede considerar como negativo sino que es un aspecto bastante trabajado e implementado en los currículos desde antes del inicio del Proyecto UNI - Barranquilla con porcentajes de cumplimiento superiores al $80 \%$ en ambas cohortes. Es de anotar que existe la necesidad de trabajar en el fortalecimiento del componente administrativo orientado hacia la solución de problemas en la comunidad y los servicios y la preparación del egresado para un eficiente desempeño corporativo.

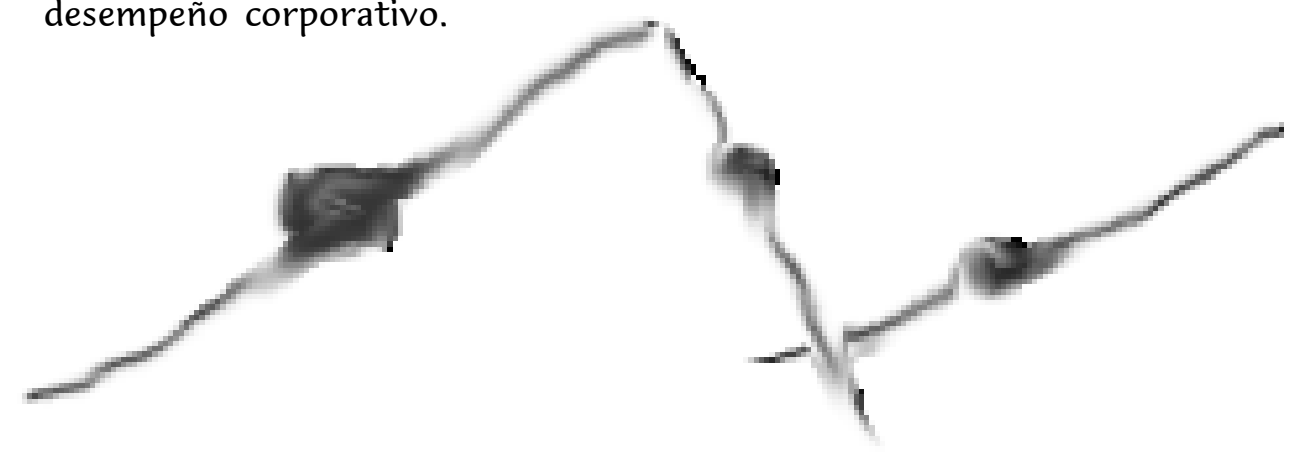


Tabla 4. Distribución de aspectos curriculares en las cohortes de estudiantes de medicina y enfermería.1993-1999. Universidad del Norte. Barranquilla

\begin{tabular}{|c|c|c|c|c|c|c|}
\hline \multirow{2}{*}{ Aspectos evaluados } & \multicolumn{2}{|c|}{ Cohorte 1993} & \multicolumn{2}{|c|}{ Cohorte 1999} & \multirow{2}{*}{ Chi cuadrado } & \multirow{2}{*}{ Valor de $p$} \\
\hline & SI & No & SI & No & & \\
\hline $\begin{array}{l}\text { Evaluación de los estudiantes centrada en } \\
\text { procesos más que en resultados }\end{array}$ & 10 & 55 & 14 & 51 & $0.81^{\star}$ & 0.36 \\
\hline $\begin{array}{l}\text { Posibilidad de desempeñarse eficientemente } \\
\text { sobre el las nuevas bases de legislación en } \\
\text { salud }\end{array}$ & 32 & 33 & 41 & 24 & $2.51^{*}$ & 0.11 \\
\hline $\begin{array}{l}\text { Fortalecimiento de los aspectos éticos en el } \\
\text { proceso de atención en las instituciones de } \\
\text { salud y en la interacción con los pacientes }\end{array}$ & 55 & 10 & 58 & 7 & $0.60^{*}$ & 0.44 \\
\hline $\begin{array}{l}\text { Componente administrativo orientado hacia } \\
\text { la solución de problemas en la comunidad y } \\
\text { los servicios }\end{array}$ & 41 & 24 & 48 & 17 & $1.73^{*}$ & 0.18 \\
\hline $\begin{array}{l}\text { Preparación para un eficiente desempeño } \\
\text { corporativo }\end{array}$ & 40 & 25 & 46 & 49 & $1.23^{*}$ & 0.27 \\
\hline
\end{tabular}

En la tabla 5, se encuentran detalladas las percepciones sobre sus habilidades y competencias reconocidas como satisfactorias durante su desempeño profesional por los egresados en las cohortes de estudiantes de medicina y enfermería. Las que mostraron diferencias estadísticamente significativas a un nivel del $95 \%$ a favor de los egresados de la cohorte del 99 son la capacidad para participar en equipos multiprofesionales, la identificación de la necesidad de buscar información para resolver un problema de salud, la capacidad de trabajar de manera conjunta con la comunidad, la identificación de la necesidad de remitir al paciente a un nivel superior de atención o consultar un profesional especialista, la capacidad para evaluar la validez de las publicaciones científicas, la capacidad para desempeñarse dentro del nuevo sistema de salud, la habilidad para realizar actividades de educación en salud, la capacidad para tomar el liderazgo del equipo de salud y la adquisición de la responsabilidad de su propio aprendizaje y aprendizaje continuo.

Tabla 5. Distribución de las percepciones sobre habilidades y competencias reconocidas como satisfactorias durante su desempeño profesional por los egresados en las cohortes de estudiantes de medicina y enfermería.1993 - 1999. Universidad del Norte. Barranquilla

\begin{tabular}{|c|c|c|c|c|c|c|}
\hline \multirow[t]{2}{*}{ Competencia o habilidad } & \multicolumn{2}{|c|}{ Cohorte 1993} & \multicolumn{2}{|c|}{ Cohorte 1999} & \multirow{2}{*}{ Chi cuadrado } & \multirow{2}{*}{ Valor de $p$} \\
\hline & SI & No & SI & No & & \\
\hline Capacidad para participar en equipos multiprofesionales & 20 & 45 & 38 & 37 & $5.64^{*}$ & 0.017 \\
\hline $\begin{array}{l}\text { Identificación de la necesidad de buscar información para resolver } \\
\text { un problema de salud }\end{array}$ & 33 & 32 & 51 & 14 & $10.82^{*}$ & 0.001 \\
\hline Capacidad de trabajar de manera conjunta con la comunidad & 42 & 23 & 53 & 12 & $4.69^{*}$ & 0.03 \\
\hline $\begin{array}{l}\text { Identificación de la necesidad de remitir al paciente a un nivel } \\
\text { superior de atención o consultar un profesional especialista }\end{array}$ & 40 & 25 & 48 & 17 & $2.23^{*}$ & 0.13 \\
\hline Capacidad para evaluar la validez de las publicaciones científicas & 31 & 34 & 49 & 16 & $10.45^{\star}$ & 0.001 \\
\hline Capacidad para desempeñarse dentro del nuevo sistema de salud & 21 & 44 & 50 & 15 & $25.9^{*}$ & 0.000004 \\
\hline Habilidad para realizar actividades de educación en salud & 45 & 20 & 56 & 9 & $4.49^{*}$ & 0.03 \\
\hline Capacidad para tomar el liderazgo del equipo de salud & 34 & 31 & 49 & 16 & $7.44^{*}$ & 0.006 \\
\hline $\begin{array}{l}\text { Responsabilidad de su propio aprendizaje y continuar el } \\
\text { autoaprendizaje }\end{array}$ & 39 & 26 & 59 & 6 & $16.45^{*}$ & 0.0000498 \\
\hline
\end{tabular}

* Estadísticamente significativo a un $95 \%$ 


\section{Discusión}

La evaluación de las modificaciones adoptadas en los programas es una necesidad indispensable para determinar el grado de impacto de las mismas. La evaluación de las innovaciones curriculares se constituye en el eje de apoyo para soportar la continuidad de los cambios y mostrar ante los diversos entes interesados el impacto de las mismas. El impacto se puede determinar en términos del estado de salud de la comunidad atendida por el grupo de egresados pero también existen otras maneras de ser medido como: los resultados del programa, los procesos educacionales adoptados y la percepción de los participantes de los programas sobre la importancia de los cambios en su desempeño profesional (McGuire, 1998; Santos Guerra, 1996; Santos, 1993; Briones, 1993).

En la presente investigación se encontró que los egresados de los programas de medicina y enfermería expuestos durante su formación académica a las innovaciones curriculares propuestas por el Proyecto UNI Barranquilla refirieron haber recibido durante su currículo una mayor integración de los contenidos básicos y profesionales, un equilibrio entre la teoría y la práctica, una diversificación de los escenarios de enseñanzaaprendizaje, una educación con orientación a los problemas más relevantes de la sociedad, realización de investigaciones acordes a las necesidades de los servicios de salud, participación en grupos de trabajo multiprofesionales, trabajo conjunto con miembros de los servicios de salud y la comunidad y una educación centrada en el alumno, como sujeto de los procesos de enseñanza-aprendizaje. Lo anterior, según la percepción de los encuestados ha logrado tener un impacto en su desempeño profesional en diferentes aspectos.

Las innovaciones curriculares promovidas por el Proyecto UNI -

Barranquilla están acordes con las directrices generales para la Educación de los Profesionales de Salud en el siglo XXI, las cuales han sido reseñadas y destacadas'en diversos escenarios y espacios de debates sobre el tema (Oberholt \& Saunders, 1996).

Aún cuando los investigadores reconocen la posibilidad de posible sesgo de información, debido al tiempo transcurrido entre la exposición a los currículos y la evaluación realizada para la cohorte de 1993, los resultados muestran que las percepciones $y$ opiniones de los egresados son congruentes con el ideario impulsado por el Proyecto UNI - Barranquilla y las innovaciones propuestas en el componente académico. $Y$ se considera que los hallazgos son un importante complemento para la evaluación de los programas y deben ser tenidos en cuenta en las nuevas propuestas de ajuste curricular que se están desarrollando en el interior de la Universidad en los currículos de Medicina y Enfermería.

\section{Conclusiones}

Los resultados muestran diferencias estadísticamente significativas entre los grupos a favor de la cohorte de egresados expuestos al ideario UNI en cuanto a la utilización de metodologías activas de enseñanzas centradas en el alumno, la posibilidad de acceder a nuevas tecnologías para fortalecer el proceso de enseñanza-aprendizaje, el fomento del trabajo en equipo 
multiprofesional con el propósito de asegurar la solución de las necesidades de la comunidad, la responsabilidad del propio aprendizaje y continuar con el proceso de autoaprendizaje después de egresado, la existencia de trabajo conjunto con líderes comunitarios, la realización de prácticas en los centros de salud $y$ en espacios comunitarios, la participación de los profesionales de los centros de salud y miembros de la comunidad en las prácticas profesionales, la realización de investigaciones acordes a la realidad epidemiológica de la región. Demuestran que las modificaciones implantadas en las estrategias de enseñanza-aprendizaje en los currículos de Medicina y Enfermería de la Universidad del Norte a través del ideario del Proyecto UNI han generado un impacto en el desempeño de los egresados en su vida profesional, aún cuando el tiempo de egresada de la cohorte expuesta al ideario es muy corta $y$ debe realizarse un estudio de seguimiento posterior.

\section{Referências}

AGUILAR, M.J.; ANDER-EGG, E. Evaluación de servicios y programas sociales. Colección Política, servicios y trabajo social. Buenos Aires: Editores LUMEN, 1994.

BARCELÓ, R.; NAVARRO, E.; YANCEN, L.M.; HERRERA, W. V.; ESPINOSA, H.; ROSALES, M.; YEPES, F. Evaluación de las concepciones, conocimientos y opiniones de los diferentes actores del proyecto UNI Barranquilla sobre el ideario UNI y el proyecto. Barranquilla: Universidad del Norte, 1996. (Documento Proyecto Uni, may - julio, 1996).

BRIONES, G. Evaluación educacional: formación de docentes en investigación educativa. 2.ed. Santa Fe de Bogotá: SECAB, 1993.

ESPINOSA TABOADA, H. Evolución del currículum de la Facultad de Medicina de la Universidad del Norte: reflexiones sobre formación integral. Barranquilla: Ediciones Uninorte, 1997.

OFICINA DE PLANEACIÓN UNIVERSIDAD DEL NORTE. Estrategias Generales de Desarrollo, 1995 1998: la Universidad hacia el siglo XXI. Santa Fe de Bogotá: Editorial Presencia, 1995.

JAUREGUI, C.A.; SUAREZ CHAVARRO, P. Promoción de la salud y prevención de la enfermedad. Enfoque en Salud Familiar. Bogotá: Editorial Médica Internacional Ltda, 1998.

MCGUIRE, C. Evaluación de programas: ¿un lujo cómodo o una necesidad básica? en educación de orientación comunitaria de los profesionales de Salud. Maastricht: Network Publications, 1998.

MURRAY, C. J. L. Cuantificación de la carga de enfermedad: la base técnica del cálculo de años de vida ajustados en función de discapacidad. Bol. Ofic. Sanit. Panam., v.118, n.3, p. 221-42, 1995.

ORDOÑEZ, M.; OCHOA, L.H.; OJEDA, G. Encuesta nacional de demografía y salud. 1995. PROFAMILIA. Santa Fe de Bogotá, 1995.

CORPOSALUD. Perfil Epidemiológico Costa Caribe Colombiana 1996: análisis de la carga de la enfermedad 1994. Colombia, 1997.

PINEDA, E. B.; ALVARADO, E. L.; CANALES, F. H. Metodología de la Investigación. 2.ed. Washington: Organización Panamericana de la Salud, 1994.

OBERHOLT, C. A.; SAUNDERS, M. K. (Eds.). Policy choices and practical problems in health economics. Washington (D.C.): World Bank, 1996.

KISIL, M.; CHAVES, M. (Orgs.) Programa UNI: una nueva iniciativa en la educación de los profesionales de la salud. Battle Creek: W. K. Kellogg Foundation, 1994. 
BARCELÓ, R.; NAVARRO, E.

SANTOS GUERRA, M. A. Evaluación educativa: un enfoque práctico de la evaluación de alumnos, profesores, centros educativos y materiales didácticos. Buenos Aires: Editorial Magisterio del Río de la Plata, 1996.

SANTOS, M. A. Hacer lo cotidiano: teoría y práctica de la evaluación cualitativa de los centros escolares. 2.ed. Madrid: Ediciones Akal, 1993.

VELASCO, J. R. Demografía social y salud pública. Cali: Universidad del Valle, 1993. (Colección Edición Previa, Serie Investigación).

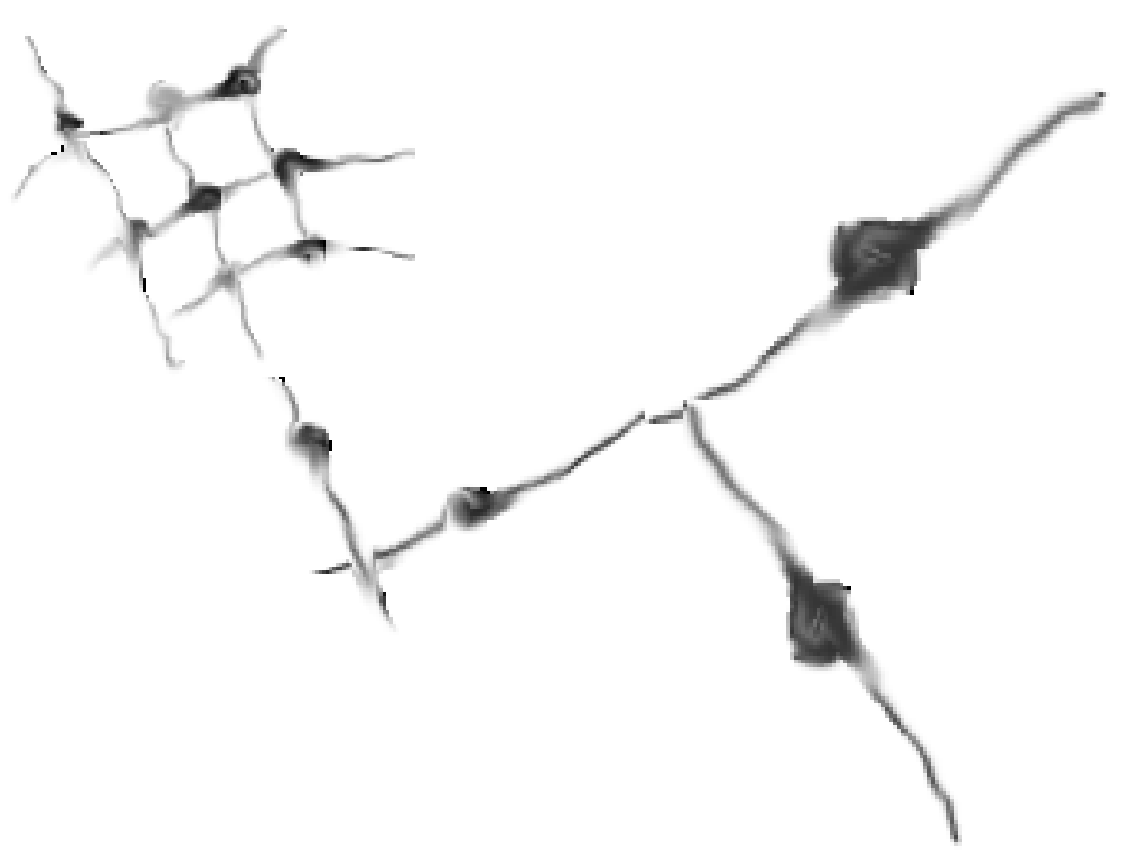

BARCELÓ, R.; NAVARRO, E. Avaliação do impacto das mudanças das metodologias de ensino-aprendizagem em egressos dos programas de Medicina e Enfermagem da Universidade do Norte, Colombia, Interface - Comunic, Saúde, Educ, v.7, n.13, p.6578, 2003.

Realizou-se um estudo descritivo transversal com o objetivo de avaliar o impacto das mudanças em metodologias de ensino-aprendizagem em estudantes dos programas de Medicina e Enfermagem da Universidade do Norte de Barranquilla, Colômbia.

Compararam-se os coortes de estudantes egressos nos anos de 1993 e 1999, estes últimos o primeiro grupo de egressos expostos às influências das mudanças curriculares propiciadas pelo Projeto Uni Barranquilla. Os dados foram obtidos mediante aplicação de questionário dirigido aos participantes. Os resultados mostram diferenças estatisticamente significativas entre os grupos, a favor do coorte de egressos expostos ao ideário Uni quanto à: utilização de metodologias ativas de ensino, possibilidade de utilizar novas tecnologias, implementar o trabalho multiprofissional e com líderes comunitários, dar continuidade ao auto-estudo, investigar a realidade epidemiológica da região e propor soluções para as necessidades apresentadas pela comunidade. Como o tempo de formação desses estudantes é muito recente, novos estudos deverão ser realizados para avaliação e seguimento posterior.

PALAVRAS-CHAVE: Estudos de avaliação; ensino; aprendizagem; métodos; currículo; universidades; Educação Médica. 